\title{
VOLUME SHIFT AND CHARGE INSTABILITY OF SIMPLE-METAL CLUSTERS
}

\author{
M. BRAJCZEWSKA, A. VIEIRA, C. FIOLHAIS \\ and J. P. PERDEW* \\ Departmento de Fsica, Universidade de Coimbra, \\ P-3000 Coimbra, Portugal \\ *Department of Physics and Quantum Theory Group, \\ Tulane University, New Orleans, LA 70118 U.S.A.
}

\begin{abstract}
Experiment indicates that small clusters show changes (mostly contractions) of the bond lengths with respect to bulk values. We use the stabilized jellium model to study the self-expansion and self-compression of spherical clusters (neutral or ionized) of simple metals. Results from Kohn - Sham density functional theory are presented for small clusters of $\mathrm{Al}$ and $\mathrm{Na}$, including negatively-charged ones. We also examine the stability of clusters with respect to charging.
\end{abstract}

\section{Introduction}

Recently, the crystallographic structure of microclusters has been the subject of many studies (see e.g. [1-7]). Standard techniques to measure the bond lengths include low energy electron diffraction, extended x-ray absorption fine structure and scanning tunneling microscopy. With few exceptions, the average interatomic distances are smaller in microclusters than in the bulk.

In the present work we use a simple theoretical model - stabilized jellium - where the ions are replaced by a continuous medium. Unlike that of the jellium model, the energy of the stabilized jellium model is stable in the bulk with respect to changes of the positive charge density. Clusters of stabilized jellium are also stable, although at a different density. Within this model, selfcompression arises from surface tension. Self-expansion can occur only for charged clusters. We shall present the model and results on self-expansion and compression which complement those already published [8-10].

\section{Model}

In the stabilized jellium model (SJM) [11], as in the jellium model, the positive ions are replaced by a continuous charge background of density $\bar{n}=3 /\left(4 \pi r_{s}^{3}\right)$, truncated sharply at a cluster radius $R_{0}=r_{s} N_{0}^{1 / 3}$, where $N_{0}$ is the number of valence electrons in the neutral cluster. 
In the SJM, the energy $E_{0}$ of a spherical neutral cluster is a function of the number of valence electrons $N_{0}$, the density parameter $r_{s}$ of the positive background, the valence $z$ and the "pseudopotential core radius" $r_{c}$. The latter is adjusted, for each metal, to achieve bulk stability at the observed $r_{s}^{B}$ (so that $r_{c}=r_{c}\left(r_{s}^{B}, z\right)$ ):

$$
\left.\lim _{N_{0} \rightarrow \infty} \frac{\partial}{\partial r_{s}}\left(E_{0} / N_{0}\right)\right|_{r_{s}=r_{s}^{B}}=0 .
$$

The number of valence electrons of a charged cluster is $N=N_{0}+\nu$ (with $\nu$ the number of excess electrons; the charge is $q=-e \nu$ ). Given the total energy of the charged cluster $E_{\nu}=E\left(N_{0}, r_{s}, z, r_{c}, \nu\right)$, the equilibrium density parameter $r_{s}^{*}$ of the cluster is obtained from:

$$
\left.\frac{\partial}{\partial r_{s}}\left(E_{\nu} / N\right)\right|_{r_{s}=r_{s}^{*}}=0
$$

The equilibrium cluster radius is then $R=r_{s}^{*} N_{0}^{1 / 3}$. We have expansion if $r_{s}^{*}>r_{s}^{B}$ or compression if $r_{s}^{*}<r_{s}^{B}$.

The elastic stiffness or inverse compressibility of the finite system is defined by :

$$
B=\left.\frac{1}{12 \pi r_{s}^{*}} \frac{\partial^{2}}{\partial r_{s}^{2}}\left(E_{\nu} / N\right)\right|_{r_{s}=r_{s}^{*}}
$$

It goes to the bulk modulus $B^{B}$ in the limit of an infinite number of particles: $\lim _{N_{0} \rightarrow \infty} B=B^{B}$.

In this work, we present $r_{s}^{*}$ and $B$ for small clusters of $\mathrm{Al}$ and $\mathrm{Na}$. We examine the role of charge in compression or dilatation, presenting new results on negatively charged clusters.

In the stabilized jellium model, the energy of the neutral or charged cluster may be evaluated by solving the self-consistent Kohn - Sham equations [12] of density functional theory. We have used the local density approximation (LDA), with the Perdew - Wang parametrization for the correlation energy [13]. These equations (in atomic units) read as

$$
\left[-\frac{1}{2} \nabla^{2}+v_{\text {eff }}(\boldsymbol{r})\right] \psi_{\alpha}(\boldsymbol{r})=\epsilon_{\alpha} \psi_{\alpha}(\boldsymbol{r})
$$

where $\alpha$ denotes a set of quantum numbers. The effective potential is

$$
v_{\mathrm{eff}}(\boldsymbol{r})=v_{+}(\boldsymbol{r})+\int d^{3} r^{\prime} \frac{n\left(\boldsymbol{r}^{\prime}\right)}{\left|\boldsymbol{r}-\boldsymbol{r}^{\prime}\right|}+\mu_{x c}(n(\boldsymbol{r})),
$$

where $v_{+}(\boldsymbol{r})$ is the electrostatic potential describing the interaction between the background and the electrons, $n(\boldsymbol{r})=\sum_{\alpha}\left|\psi_{\alpha}(\boldsymbol{r})\right|^{2} \Theta\left(\mu-\epsilon_{\alpha}\right)$ is the electronic density, with $\mu$ the chemical potential, and $\mu_{x c}(n(\boldsymbol{r}))$ the exchange-correlation potential. We have [14]

$$
v_{+}(\boldsymbol{r})= \begin{cases}-\frac{N}{2 R}\left[3-\left(\frac{r}{R}\right)^{2}\right]+\langle\delta v\rangle_{W S} & (r<R) \\ -\frac{N}{r} & (r>R)\end{cases}
$$

with

$$
\langle\delta v\rangle_{W S}=-\frac{1}{5}\left(\frac{9 \pi}{4}\right)^{2 / 3} \frac{r_{s}^{B}}{r_{s}^{3}}+\frac{1}{4 \pi}\left(\frac{9 \pi}{4}\right)^{1 / 3} \frac{\left(r_{s}^{B}\right)^{2}}{r_{s}^{3}}+\frac{1}{3} \frac{\left(r_{s}^{B}\right)^{4}}{r_{s}^{3}} \frac{d \epsilon_{c}\left(r_{s}^{B}\right)}{d r_{s}^{B}}+\frac{3}{10}\left(\frac{\left(r_{s}^{B}\right)^{2}}{r_{s}^{3}}-\frac{1}{r_{s}}\right)
$$

the constant potential of the stabilized jellium model. The exchange-correlation potential in the LDA is given by $\mu_{x c}=\frac{\partial}{\partial n}\left[n \epsilon_{x c}\right]$, with $\epsilon_{x c}(n)$ the exchange-correlation energy per electron for an electron gas of uniform density $n$. 
Once Eq. (4) has been solved, the total energy of the cluster is given by:

$$
E[n(\boldsymbol{r})]=\sum_{\alpha} \epsilon_{\alpha}-U[n]+U_{B}\left[n_{+}\right]-\int d^{3} r n(\boldsymbol{r}) \mu_{x c}(n(\boldsymbol{r}))+\int d^{3} r n(\boldsymbol{r}) \epsilon_{x c}(n(\boldsymbol{r}))
$$

where

$$
U[n]=\frac{1}{2} \int d^{3} r \int d^{3} r^{\prime} \frac{n(\boldsymbol{r}) n\left(\boldsymbol{r}^{\prime}\right)}{\left|\boldsymbol{r}-\boldsymbol{r}^{\prime}\right|}
$$

is the electronic Coulomb repulsion energy, and $U_{B}\left[n_{+}\right]=3 N_{0}\left(N_{0}-N_{0}^{1 / 3}\right) / 5 R$ is the background Coulomb repulsion minus the self-repulsion energy within each Wigner-Seitz cell.

The energy of a charged cluster may also be evaluated using the liquid drop model (LDM), which disregards shell fluctuations and therefore provides a continuous description of the energy which is useful to identify trends $[15,16]$. This energy is

$$
E_{\nu}=E_{0}-\nu\left(W+\frac{c}{R}\right)+\frac{\nu^{2}}{2(R+\delta)},
$$

with $E_{0}$ the energy of the neutral cluster, $W$ the work function, $c$ a parameter measuring the first-order size correction to the work function, and $\delta$ the distance from the image plane to the jellium edge of the planar surface. The energy $E_{0}$ is given by

$$
E_{0}=a_{v}\left(r_{s}, z, r_{c}\right) N_{0}+a_{s}\left(r_{s}, z, r_{c}\right) N_{0}^{2 / 3}+a_{c}\left(r_{s}, z, r_{c}\right) N_{0}^{1 / 3},
$$

where $a_{v}$ is the average energy per electron in the bulk system, $a_{s}$ the surface and $a_{c}$ the curvature coefficients. These coefficients may be extracted from the planar surface problem with the aid of the so-called "leptodermous expansion" [17,18].

\section{Results}

For $\mathrm{Al}$ and $\mathrm{Na}$, with bulk density parameters respectively $r_{s}^{B}=2.07$ and 3.93 bohr and valences $z=3$ and 1 , the following conclusions were drawn in previous works [8-10]:

Surface tension compresses the neutral cluster (i.e., $r_{s}^{*}<r_{s}^{B}$ ). Kohn - Sham calculations display shell-structure oscillations around the average behaviour predicted by the LDM and produce local minima in the density parameter $r_{s}^{*}$ and local maxima in the elastic stiffness $B$ for closed-shell clusters $N=2,8,18,20$ etc..

In charged clusters two effects compete: surface tension increases the ionic density, while surface-charge Coulomb repulsion reduces it. Self-expansion occurs when the Coulomb repulsion of the excess charge overwhelms the surface tension. For very large charges, explosion arises via disappearance of a solution for the background equilibrium radius.

Let us now examine the compression or expansion effects for a broader range of charges. Charged clusters of aluminum, with charges between $q=-2$ and $q=+2$ show self-compression. Fig. 1 shows how the equilibrium radius of an aluminum cluster depends upon the number of 
atoms $N_{\text {at }}$ (for Al, $N_{\text {at }}=N_{0} / 3$ ) and the charge $q$. A shell structure (with minima in $r_{s}^{*}$ for electron numbers around 18 and 40 ) is superimposed on a smooth pattern of self-compression, which is stronger for small systems than for large, and stronger for positive charges than for neutral or negative ones.

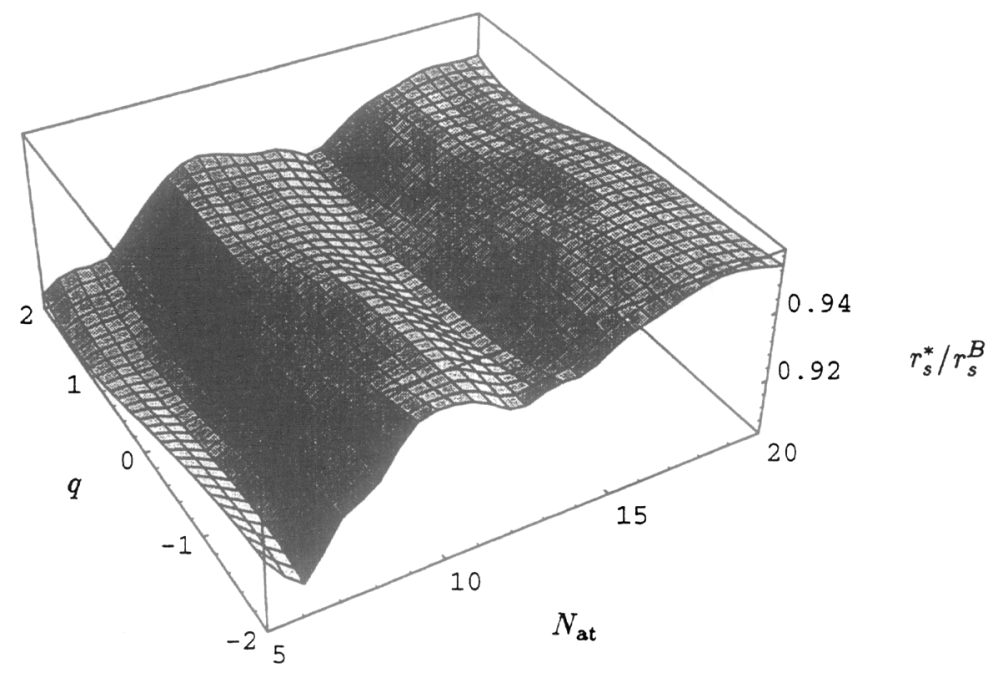

Fig. 1. Equilibrium density parameter $r_{s}^{*}$ of aluminum clusters with $N_{\text {at }}=5, \ldots, 20$ and charges $q=-2, \ldots, 2$. The LDA Kohn - Sham values for each $N_{\text {at }}=N_{0} / z$ and $q$ were evaluated in the stabilized jellium model, and a surface was fitted to these results.

Bigger charges approach the limits of stability. Consider the magic system $\mathrm{Al}_{6}$ which, from atomistic studies, is known to be the smallest $\mathrm{Al}$ cluster with a compact shape. If we ionize this cluster, extracting electrons, there is no solution for the background equilibrium density for charges $q$ equal to or bigger than 12, while the transition from self-compression to self-expansion takes place at $q=6$ (Fig. 2). On the other hand, if we ionize the non-magic system $\mathrm{Al}_{8}$ we see that the system can subsist up to $q=13$. Thus it appears that the size of the cluster, and not the shell structure, primarily sets the limit of stability with respect to Coulomb explosion.

From the elastic stiffness, $\mathrm{Al}_{6}^{0}$ is somewhat harder than $\mathrm{Al}_{8}^{0}$. (Both are harder than the bulk, so that a crystal made of packed Al clusters might have a larger bulk modulus than normal Al). The elastic stiffness for small charges is independent of the charge, but the "explosion" of the system is "announced" by the vanishing of the stiffness. The instability which is being analyzed is different from that associated with cluster fission, namely instability with respect to deformation holding the volume fixed. In our present work the volume is allowed to change while the system keeps a spherical shape. More charge is needed to blow-up the volume than to deform the shape and fragment the cluster [19]. 

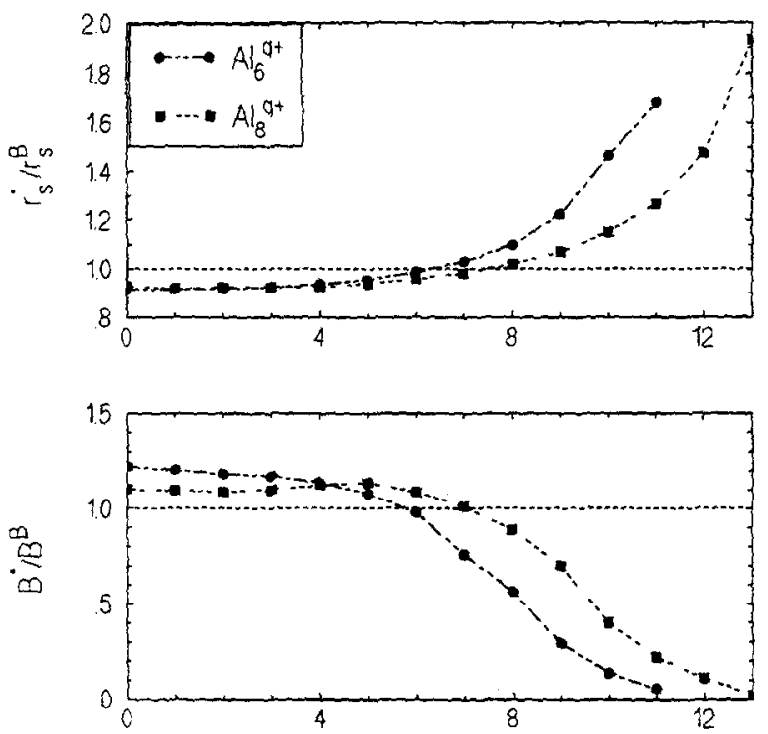

a

Fig. 2. Above: Equilibrium density parameter for $\mathrm{Al}_{6}^{9+}$ and $\mathrm{Al}_{8}^{q+}$ as a function of the positive charge $q$. (This graph is a cut of Fig. 1 for $N_{\mathrm{at}}=6$ and 8). For $q=0$ the first cluster is magic, while the second is not. Below: Elastic stiffness for the same systems.
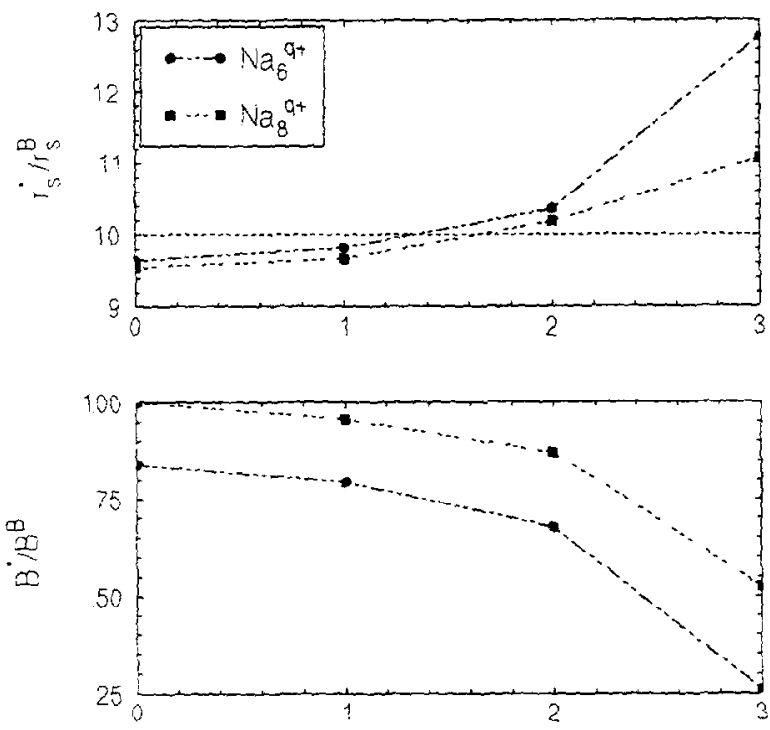

q

Fig. 3. Same as Fig. 2, for $\mathrm{Na}_{6}^{q+}$ and $\mathrm{Na}_{8}^{q+}$. Note that for $q=0$ the first cluster is not magic while the second is. 

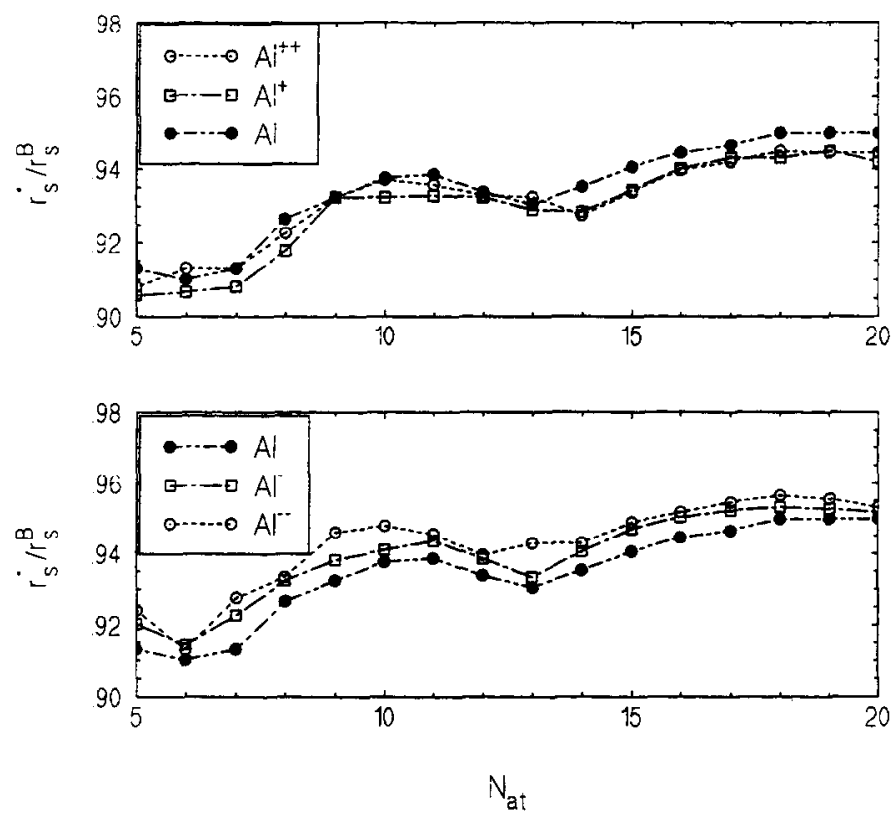

Fig. 4. Above: Equilibrium density of neutral, single and double positively charged clusters of aluminum as a function of the number of atoms. (This graph is a cut of Fig. 1 for $q=0, \pm 1$ ). Below: Same as above for negatively charged clusters.
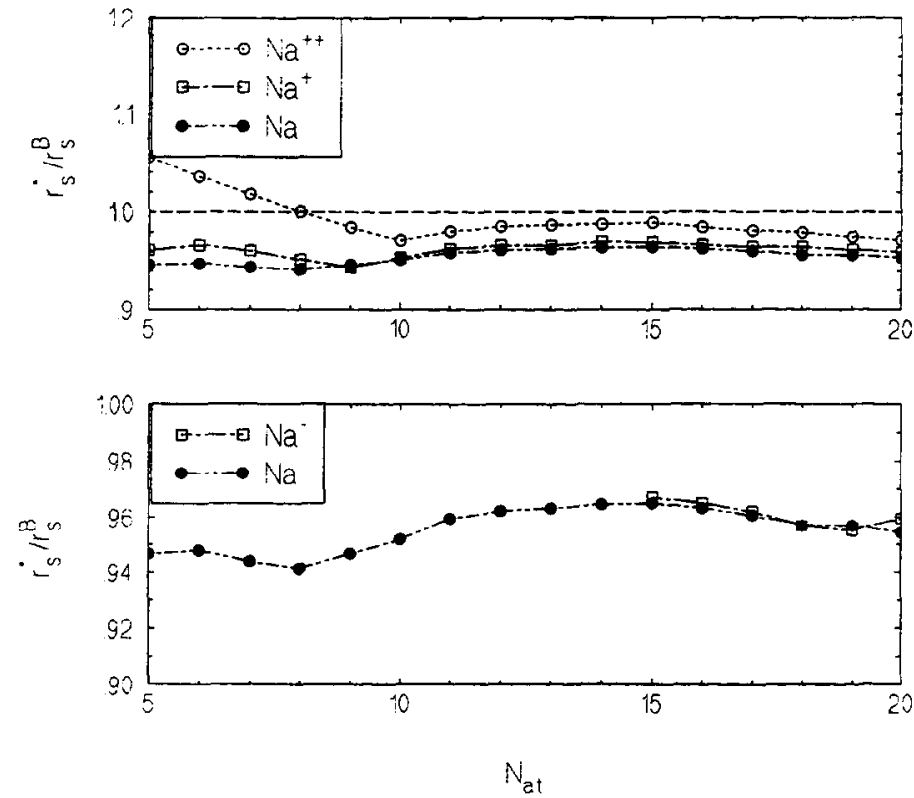

Fig. 5. Same as Fig. 4, for sodium. There are no bound solutions of the Kohn - Sham equations for $\mathrm{Na}^{-}$up to $N_{\text {at }}=15$. 
In Fig. 3 we see similar effects for $\mathrm{Na}$, the difference being that $\mathrm{Na}$ clusters with the same number of atoms are not able to sustain charges as big as in Al. Self-expansion occurs for $q \geq 2$, and the limits of stability occur at $q=4$, in both $\mathrm{Na}$ clusters.

It is expected [10] that neutral clusters will show a stronger self-compression than charged clusters of the same size, and that highly-charged clusters will display self-expansion. Figs. 4 and 5 show that this expectation is met by $\mathrm{Na}$ clusters, and by negative Al clusters, but that $\mathrm{Al}$ clusters with small positive charges are anomalously (but only slightly) more compressed than neutral ones. Fig. 6 displays the asymmetry between positive and negative charges for the equilibrium radii of $\mathrm{Al}_{8}$ clusters. We also show the difference between $\mathrm{Al}_{8}^{q}$ and $\mathrm{Na}_{24}^{q}$, which have the same number of electrons. The LDM result is superimposed on the Kohn - Sham result, and does not exhibit the anomalous behavior of the latter.
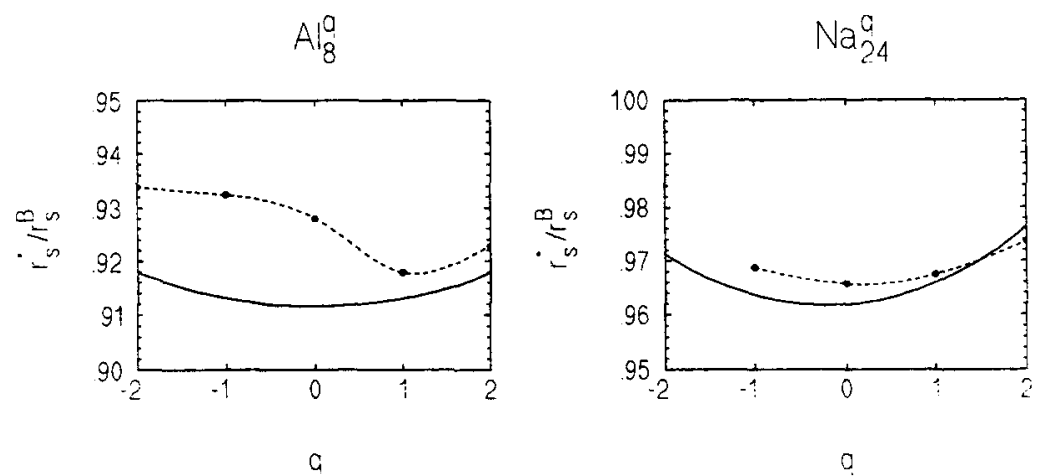

Fig. 6. Left: Equilibrium density parameter of $\mathrm{Al}_{8}^{q}$ as a function of the charge. Liquid drop results (full line) are compared with the quantal ones. (The dashed line is a spline which connects the calculated points and guides the eye). Right: The same for $\mathrm{Na}_{24}^{q}$.

LDA fails to find self-consistent solutions for small negative ions [20]. For aluminum, the smallest singly-negative ion for which we find a solution is $\mathrm{Al}_{2}^{-}$, and the smallest doubly-negative ion is $\mathrm{Al}_{4}^{--}$. For sodium, the smallest singly-negative ion for which we find a solution is $\mathrm{Na}_{15}^{-}$. However, this does not mean that LDA predicts no smaller negative sodium ion, as we discuss in the next paragraph.

The liquid drop model can be used to discuss the stability of negatively-charged Na clusters against emission of an electron. To simplify the discussion, replace $c / R$ by $c /(R+\delta)$ in Eq. (10). Then a neutral cluster with $N_{0}$ valence electrons can bind $\nu$ excess electrons when $E_{\nu-1}>E_{\nu}$, i.e., $[10]$

$$
N_{0}>\left[\frac{\nu-c-\frac{1}{2}}{W r_{s}^{*}}-\frac{\delta}{r_{s}^{*}}\right]^{3}
$$


Eq. (12) predicts that $\mathrm{Na}_{2}$ and larger sodium clusters can lower their energy by binding one excess electron. However, the condition for a self-consistent solution of the Euler ( $\sim$ Kohn Sham) equation is $\mu \equiv d E_{\nu} / d \nu<0$, i.e.

$$
N_{0}>\left[\frac{\nu-c}{W r_{s}^{*}}-\frac{\delta}{r_{s}^{*}}\right]^{3}
$$

Eq. (13) predicts that only $\mathrm{Na}_{13}$ and larger clusters should have self-consistent solutions that bind one excess electron, in reasonable agreement with our Kohn - Sham results of Fig. 5. According to both the LDM and the Kohn - Sham LDA, singly-negative clusters from $\mathrm{Na}_{2}^{-}$to $\mathrm{Na}_{12}^{-}$are stable with respect to removal of a whole electron, but unstable with respect to removal of a fraction of an electron.

\section{Conclusions}

Simple theory based on the concept of surface tension predicts that neutral clusters should self-compress, i.e., that the bond lengths of a cluster should be smaller than in the bulk. We have called attention to the role of charge, including negative charge. In the SJM, the charging properties of $\mathrm{Al}$ and $\mathrm{Na}$ clusters are different: Aluminum clusters with the same number of particles may sustain a much bigger charge than sodium clusters. Moreover, the charge dependence of the equilibrium radius of $\mathrm{Al}$ and $\mathrm{Na}$ with the same number of particles is different: $\mathrm{Al}$ clusters do not show a minimum for the neutral system. As the magnitude of the charge increases, of course, any system should become less dense than the bulk and should eventually break up.

\section{Acknowledgements}

This work has been partially supported by the Praxis XXI Program (Project 2/2.1/FIS/26/94) and by the Junta Nacional de Investigao Cientfica e Tecnolgica (Project PBIC/C/FIS/2216/95). One of us (A.V.) was supported by Praxis XXI Grant and one of us (J.P.P.) by the U.S. National Science Foundation, Grant DMR 95-21353.

\section{References}

[1] P.A. Montano, J. Zhao, M. Ramanathan, G.K. Shenoy, W. Schulze and J. Urban, Chem. Phys. Lett. 164, 126 (1989).

[2] K. Heinemann and H. Poppa, Surface. Sci. 156, 265 (1985).

[3] J. Forges, M.F. de Feraudy, B. Raoult and G. Torchet, J. Chem. Phys. 78, 5067 (1983).

[4] D.W. Abraham, K. Sattler, E. Ganz, H.J. Mamin, R.E. Thomson and J. Clarke, Appl. Phys. Lett. 49, 853 (1986).

[5] S. Iijima and T. Ichihashi, Phys. Rev. Lett. 56, 616 (1986).

[6] A. Balerna, E. Bernieri, P. Picozzi, A. Reale, S. Santucci, E. Burattini and S. Mobilio, Surface Sci.156, 206 (1985).

[7] G. Apai, J.F. Hamilton, J. Stohr and A. Thompson. Phys. Rev. Lett. 43, 165 (1979).

[8] J.P. Perdew, M. Brajczewska and C. Fiolhais, Solid State Commun. 88, 795 (1993).

[9] M. Brajczewska, C. Fiolhais, A. Vieira and J.P. Perdew, in Many-Body Physics, C. Fiolhais et al. (Eds.), World Scientific, Singapore (1994), p. 455. 
[10] A. Vieira, C. Fiolhais, M. Brajczewska and J.P. Perdew, Int. J. Quantum Chem., in print.

[11] J.P. Perdew, H.Q. Tran and E.D. Smith, Phys. Rev. B 42, 11627 (1990).

[12] W. Kohn and L.J. Sham, Phys. Rev. 140, A1133 (1965).

[13] J.P. Perdew and Y. Wang, Phys. Rev. B 45, 13244 (1992).

[14] M. Brajczewska, C. Fiolhais and J.P. Perdew, Int. J. Quantum Chem. S 27, 249 (1993).

[15] J.P. Perdew, in Condensed Matter Thecries, J. Keller (Ed.), Plenum, New York (1989), vol. 4.

[16] M. Seidl and M. Brack, Annals of Physics (N.Y.) 245, 275 (1996); M. Seidl and J.P. Perdew, Phys. Rev. B 50, 5744 (1994).

[17] M. Brack, C. Guet and H.-B. Hakansson, Phys. Rep. 123, 275 (1985).

[18] C. Fiolhais and J.P. Perdew, Phys. Rev. B 45, 6207 (1992).

[19] A. Vieira, M. Brajczewska and C. Fiolhais, Int. J. Quantum Chem. 56, 239 (1995).

[20] A. Rubio, L.C. Balbas and J.A. Alonso, Physica B 168, 32 (1991). 